\title{
Comparison between 3D-IR-BTFE Method and Conventional Method in Contrast Head MRI Examination*
}

\author{
Daisuke Hirahara \\ Department of Medical Technology, Medical Professional College, Kagoshima, Japan \\ Email: ffieldai@gmail.com
}

How to cite this paper: Hirahara, D. (2018) Comparison between 3D-IR-BTFE Method and Conventional Method in Contrast Head MRI Examination. Open Journal of Medical Imaging, 8, 31-40. https://doi.org/10.4236/ojmi.2018.83005

Received: July 25, 2018

Accepted: August 25, 2018

Published: August 28, 2018

Copyright $\odot 2018$ by author and Scientific Research Publishing Inc. This work is licensed under the Creative Commons Attribution International License (CC BY 4.0).

http://creativecommons.org/licenses/by/4.0/

\begin{abstract}
MRI using gadolinium contrast media is useful in diagnosis; however, nephrogenic systemic fibrosis is a serious side effect of gadolinium exposure. Moreover, it turns out that gadolinium deposits in the brain. This has escalated the necessity for a suitable method to use gadolinium contrast media. I developed a new imaging method that had excellent contrast. This study examined the usefulness of that new imaging method and found the method is highly effective.
\end{abstract}

\section{Keywords}

Gadolinium, BTFE, Brain, MRI, II Nversion Recovery, T1 Weighted Image, Spin Echo, Gradient Echo

\section{Background}

Exact diagnosis of a metastatic brain tumor is important for stage judging and future treatment policy. The first choice for metastatic brain tumor imaging is MRI, which uses double the amount of gadolinium contrast media [1] [2] [3] [4]. MRI using gadolinium contrast media plays an important role in the differential diagnosis of other brain tumors. However, it has been reported that nephrogenic systemic fibrosis (NSF) is a serious side effect of gadolinium contrast media. One cause of NSF is that contrast media chemical structure is not a macro ring contexture. Another cause is the amount used. Therefore, a renal function check is now required. Moreover, the amount used and the interval of contrast media were optimized. Kanda et al. 2013 showed that with a contrast media that is not a macro ring contexture, the risk to a brain is clear, and did not

*This research paper is pre-reprinted by Bioaxiv. 
recommend the use of any contrast media other than those with a macro ring contexture. Subsequent research revealed that macro ring contexture agents also deposited slightly in the brain. To avoid risks, it is necessary to use gadolinium contrast media appropriately [5] [6] [7]. After gadolinium imaging, a T1 image of the spin echo method was used. The three-dimensional gradient echo imaging method (3D-GRE) T1 image was also used. The MPRAGE method was used in $3 \mathrm{~T}$ by the influence of SAR and T1 extension [8].

\section{Purpose}

With an imaging method that emphasizes the contrast effect more than the conventional imaging method, there is a high possibility of reducing the risk of side effects due to the reduction of the gadolinium contrast agent. Further, there is a high possibility of improving the lesion detection rate due to the increase in contrast. In this study, 3D-IR (IR) and the inversion recovery method (IR) were combined to obtain a T1 emphasis based on a balanced turbo field echo (BTFE) sequence, which is a coherent gradient echo method, which is a high signal-to-BTFE imaging method was prepared and the conventional imaging method and contrast were examined [9].

\section{Apparatus and Method}

This survey was conducted in accordance with the Declaration of Helsinki; eights subjects orally consented. Eight subjects were all men in their 70s.There are eight examples of metastatic brain tumor searches. The equipment was a superconducting 1.5 Tesla MRI machine (Intera Achieva Nova, PHILIPS) using an $8 \mathrm{CH}$ SENSE head coil. The contrast media used was gadolinium (HP-DO 3A) $(0.4 \mathrm{ml} / \mathrm{kg})$ for the patient. EZR was used for statistical processing [10]. I used a slice thickness of $3 \mathrm{~mm}$ of T1 emphasis picture (SE-T1WI) for the two-dimensional spin echo method and a slice thickness of $1 \mathrm{~mm}$ for the three-dimensional gradient echo method (3D-GRE). 3D-IR-BTFE also used a slice thickness of $1 \mathrm{~mm}$. I reconstructed in a slice thickness of $3 \mathrm{~mm}$ so that SE-T1WI could be compared with an imaging method with a slice thickness of $1 \mathrm{~mm}$. To eliminate the order effect, the order of each imaging method after gadolinium imaging was random. Considering the distribution of gadolinium contrast media in the brain, I started the image pick-up 5 minutes after pouring the contrast media. The main imaging conditions are shown in Table 1.

This evaluation compared contrast. Contrast comparison set the area of interest (ROI) as a pathological change in brain substance and evaluated it.

For the statistical analysis, a Wilcoxon signed-rank test was performed. ${ }^{\star *}$ of statistical analysis result indicates $\mathrm{p}<0.01$.

\section{Result}

Part of an obtained image is shown in Figure 1. The yellow circle shown in Figure 1 is an example of a metastatic lesion. It can be seen that the contrast varies 
Table 1. Shows the main imaging parameters of the SE-T1WI \& 3D-GRE \& 3D-IR-BTFE.

\begin{tabular}{|c|c|c|c|c|c|}
\hline \multicolumn{2}{|c|}{ SE-T1WI parameters } & \multicolumn{2}{|c|}{ 3D-GRE parameters } & \multicolumn{2}{|c|}{ 3D-IR-BTFE parameters } \\
\hline FOV & 230 & FOV & 240 & FOV & 230 \\
\hline RFOV & 80 & RFOV & 100 & RFOV & 80 \\
\hline Matrix & 256 & Matrix & 240 & Matrix & 224 \\
\hline Recon & 512 & Recon & 256 & Recon & 256 \\
\hline Scan \% & 70 & Scan $\%$ & 78.54 & Scan $\%$ & 110 \\
\hline SENSE & No & SENSE & yes & SENSE & Yes \\
\hline Slices & 48 & Slices & 175 & Slices & 140 \\
\hline Slice thickness & 3 & Slice thickness & 1 & Slice thickness & 1 \\
\hline Slice gap & 0 & Slice gap & 0 & Slice gap & 0 \\
\hline Scan mode & MS & Scan mode & $3 \mathrm{D}$ & Scan mode & $3 \mathrm{D}$ \\
\hline Technique & SE & Technique & FFE & Technique & FFE \\
\hline $\mathrm{TR}$ & 462 & TR & 25 & TR & 4.3 \\
\hline $\mathrm{TE}$ & 15 & $\mathrm{TE}$ & 4.6 & $\mathrm{TE}$ & 2.2 \\
\hline Flip angle & 90 & Flip angle & 30 & Flip angle & 60 \\
\hline NSA & 2 & NSA & 1 & NSA & 2 \\
\hline \multirow[t]{5}{*}{ Scan time } & $6: 48$ & Scan time & $4: 52$ & Scan time & $3: 38$ \\
\hline & & P reduction & 2 & $\mathrm{P}$ reduction & 2.5 \\
\hline & & $S$ reduction & 2 & $S$ reduction & 1 \\
\hline & & & & TFE factor & 256 \\
\hline & & & & Shot interval & 4000 \\
\hline
\end{tabular}

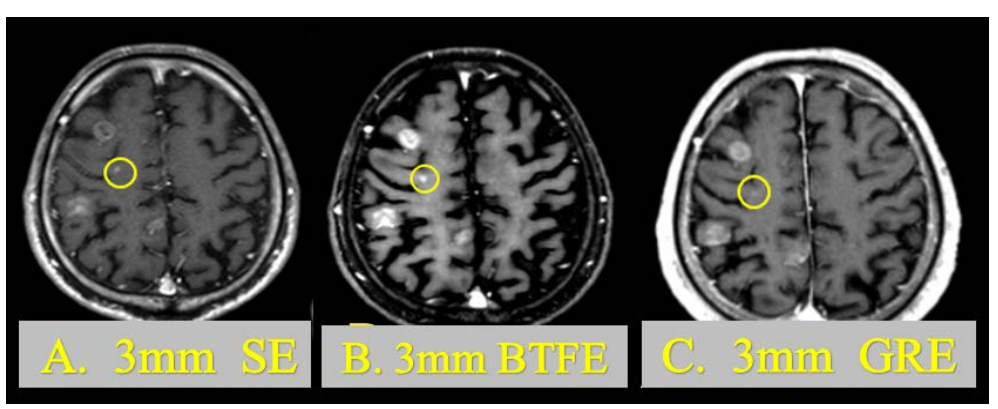

Figure 1. Shows a photographed image. A is SE-T1WI of $3 \mathrm{~mm}$ after imaging. $\mathrm{B}$ is 3D-IR-BTFE of $3 \mathrm{~mm}$ after imaging. $\mathrm{C}$ is $3 \mathrm{D}$-IR-BTFE of $1 \mathrm{~mm}$ after imaging.

with the imaging method.

Figure 2 shows an example of ROI measurement. Measurements were conducted so that all ROI sizes are the same.

An example of a setting a ROI is shown in Figure 2.

The difference in the average values of SE-T1WI and 3D-IR-BTFE is shown in Figure 3. The difference test of the average value was $t(19)=-8.252, p<0.01$ ( $p$ $=0.0000001), \mathrm{d}=-9.64$. 


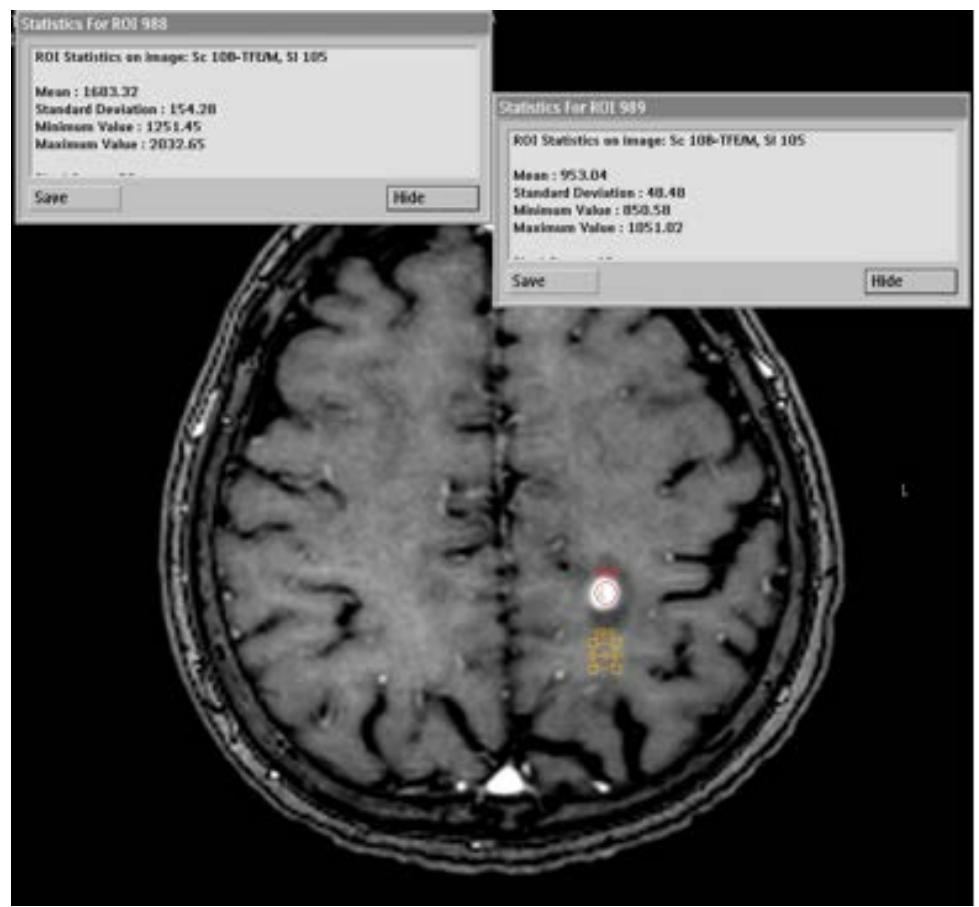

Figure 2. Shows a method for setting the actual region of interest.

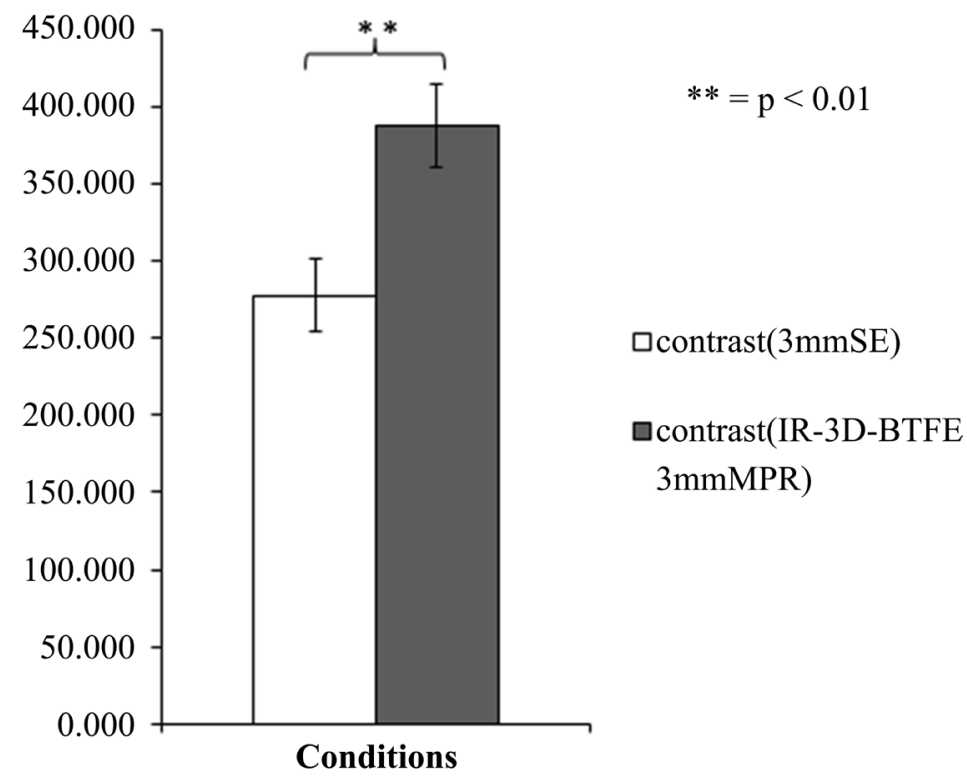

Figure 3. Shows the difference between the average values of SE-T1WI and 3D-IR-BTFE (slice thickness $3 \mathrm{~mm}$ ).

The difference of the average value of slice thickness $1 \mathrm{~mm}$ of SE-T1WI and 3D-IR-BTFE is shown in Figure 4. The difference test of the mean value was $t$ $(19)=-10.828, p<0.01(p=0.0000000014), d=-1.656$.

The difference of the average value of 3D-IR-BTFE $3 \mathrm{~mm}$ and 3D-IR-BTFE $1 \mathrm{~mm}$ is shown in Figure 5. The difference test of the average value was $\mathrm{t}(19)=$ $-5.637, \mathrm{p}<0.01(\mathrm{p}=0.000019), \mathrm{d}=-787$. 


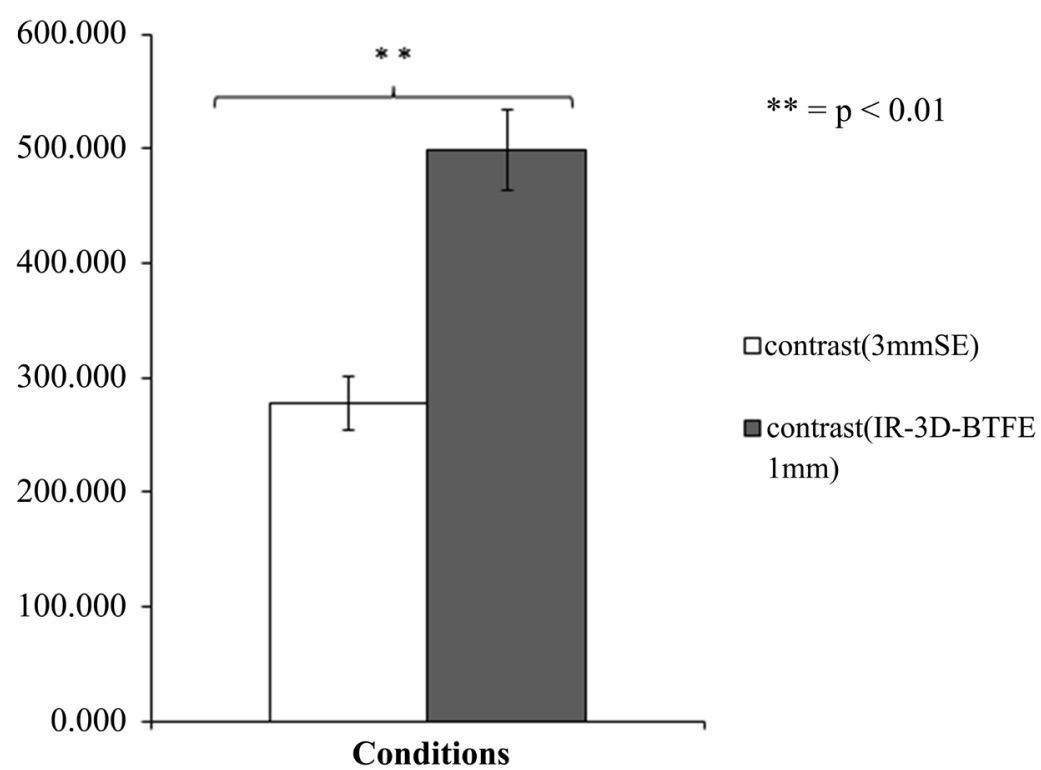

Figure 4. Shows the differences between average values of SE-T1WI and 3D-IR-BTFE (slice thickness $1 \mathrm{~mm}$ ).

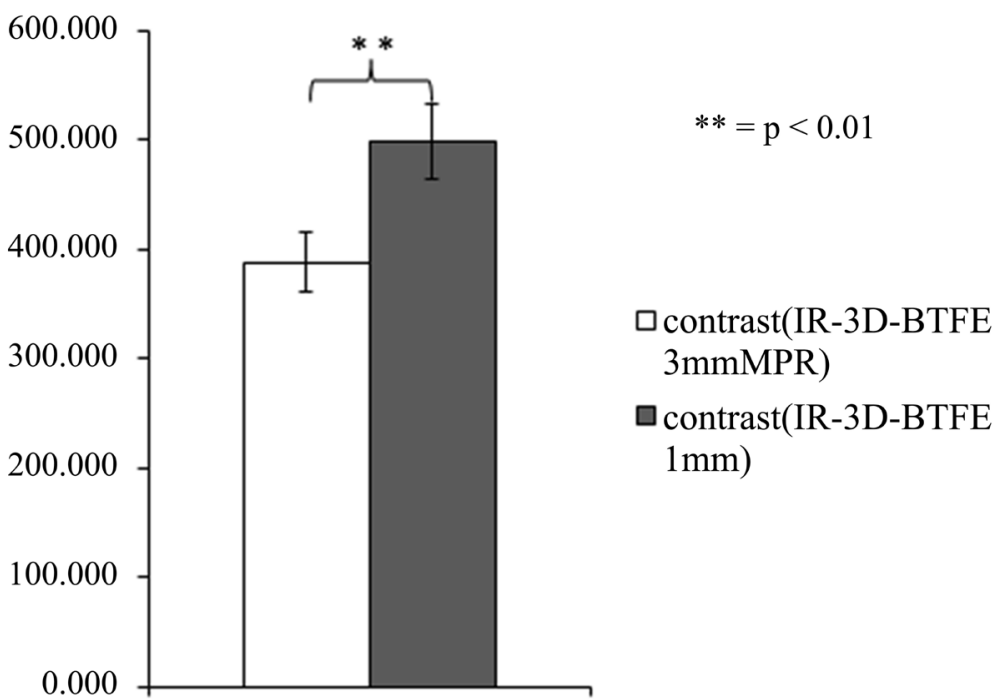

\section{Conditions}

Figure 5. Shows the difference between the average value of 3D-IR-BTFE (slice thickness $3 \mathrm{~mm}$ ) and 3D-IR-BTFE (slice thickness $1 \mathrm{~mm}$ ).

The difference between the slice thickness of $3 \mathrm{~mm}$ of SE-T1WI and IR-3 D-BTFE and the average of the ranks of IR-3 D-BTFE $1 \mathrm{~mm}$ (Friedman examination) is shown in Figure 6. The difference test was $\mathrm{z}(14)=36.100, \mathrm{p}<0.01(\mathrm{p}$ $=0.00000001), \mathrm{r}=0.602$.

The 3D-IR-BTFE method gave an average higher contrast result than SE-T1WI. The results are shown in Table 2.

The contrast results of 3D-GRE and 3D-IR-BTFE in 12 lesions are shown in Table 3. 


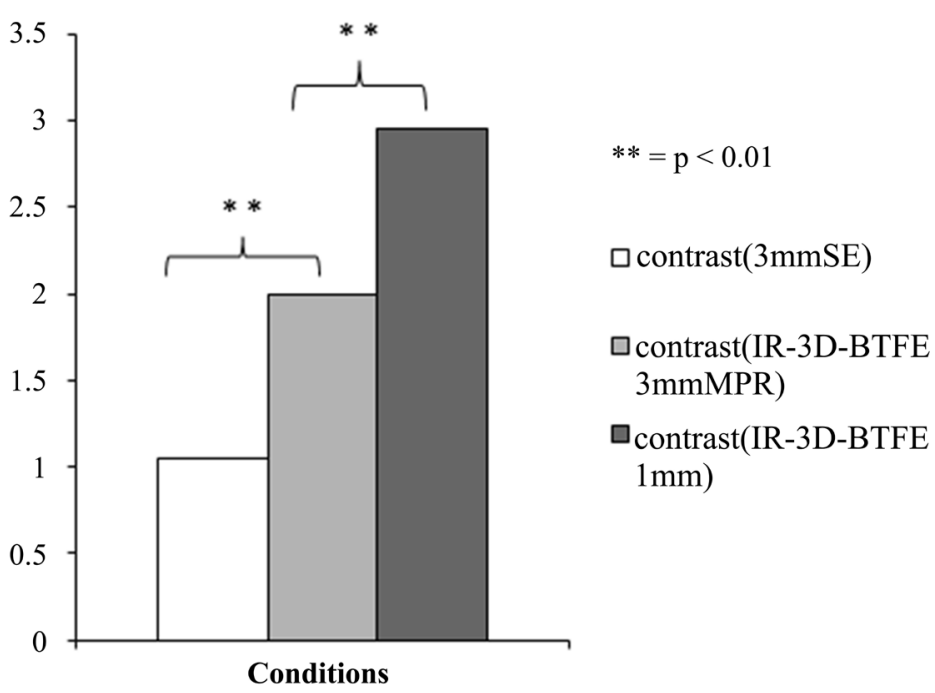

Figure 6. Shows the results of Friedman's test of the difference between SE-T1WI and 3D-IR-BTFE (slice thickness $3 \mathrm{~mm}$ ) and 3D-IR-BTFE (slice thickness $1 \mathrm{~mm}$ ).

Table 2. Shows a comparison of contrast between 3D-IR-BTFE and SE-T1WI.

\begin{tabular}{|c|c|c|c|c|c|c|c|c|c|}
\hline & \multicolumn{3}{|c|}{$3 \mathrm{~mm} \mathrm{SE}$} & \multicolumn{3}{|c|}{ IR-BTFE $3 \mathrm{~mm}$ MPR } & \multicolumn{3}{|c|}{ IR-BTFE $1 \mathrm{~mm}$} \\
\hline & Leision & Brain & Contrast & Leision & Brain & Contrast & Leision & Brain & Contrast \\
\hline 1 & 767.59 & 504.1 & 263.49 & 1194.65 & 732.48 & 462.17 & 1241.48 & 706.61 & 534.87 \\
\hline 2 & 818.6 & 547.67 & 270.93 & 1274.98 & 835.44 & 439.54 & 1394.85 & 839.19 & 555.66 \\
\hline 3 & 802.47 & 502.89 & 299.58 & 1159.52 & 679.9 & 479.62 & 1210.07 & 691.3 & 518.77 \\
\hline 4 & 1017 & 520.36 & 496.64 & 1498.92 & 957.27 & 541.65 & 1683.32 & 953.04 & 730.28 \\
\hline 5 & 844.94 & 516.37 & 328.57 & 1365.81 & 977.68 & 388.13 & 1635.77 & 985.81 & 649.96 \\
\hline 6 & 735.72 & 516.37 & 219.35 & 1291.14 & 977.68 & 313.46 & 1514.3 & 985.81 & 528.49 \\
\hline 7 & 726.35 & 486.47 & 239.88 & 1318.84 & 946.68 & 372.16 & 1339.56 & 899.97 & 439.59 \\
\hline 8 & 801.8 & 480.65 & 321.15 & 1216.07 & 774.04 & 442.03 & 1213.19 & 761.98 & 451.21 \\
\hline 9 & 811.22 & 531.24 & 279.98 & 1082.89 & 750.08 & 332.81 & 1189.34 & 688.42 & 500.92 \\
\hline 10 & 809.4 & 521.76 & 287.64 & 1227.62 & 795.68 & 431.94 & 1202.55 & 797.5 & 405.05 \\
\hline 11 & 666.41 & 521.76 & 144.65 & 1034.51 & 795.68 & 238.83 & 1068.88 & 797.5 & 271.38 \\
\hline 12 & 687.53 & 549.74 & 137.79 & 1006.08 & 776.77 & 229.31 & 1149.89 & 796.15 & 353.74 \\
\hline 13 & 797.74 & 569.01 & 228.73 & 1189.14 & 870.76 & 318.38 & 1269.73 & 862.68 & 407.05 \\
\hline 14 & 565.29 & 538.04 & 27.25 & 903.09 & 835.41 & 67.68 & 940.13 & 847.72 & 92.41 \\
\hline 15 & 801.79 & 493.68 & 308.11 & 1064.89 & 784.71 & 280.18 & 1187.61 & 752.45 & 435.16 \\
\hline 16 & 774.31 & 493.68 & 280.63 & 1189.78 & 784.71 & 405.07 & 1217.95 & 752.45 & 465.5 \\
\hline 17 & 849.28 & 485.54 & 363.74 & 1296.03 & 809.89 & 486.14 & 1557.66 & 779.61 & 778.05 \\
\hline 18 & 946.97 & 476.38 & 470.59 & 1339.57 & 781.06 & 558.51 & 1415.77 & 791.63 & 624.14 \\
\hline 19 & 912.22 & 560.43 & 351.79 & 1434.4 & 878.8 & 555.6 & 1506.11 & 897.93 & 608.18 \\
\hline 20 & 711.89 & 473.89 & 238 & 1154.17 & 736.36 & 417.81 & 1291.93 & 674.3 & 617.63 \\
\hline Average & 792.426 & 514.5 & 277.926 & 1212.11 & 824.05 & 388.06 & 1311.5 & 813.1 & 498.4 \\
\hline
\end{tabular}


Table 3. Shows a comparison of contrast between 3D-IR-BTFE and SE-T1WI.

\begin{tabular}{|c|c|c|c|c|c|c|c|}
\hline & \multicolumn{3}{|c|}{ 3D-IR-BTFE } & \multicolumn{3}{|c|}{ 3D-GRE } & \multirow{2}{*}{$\begin{array}{c}\text { Contrast } \\
\text { BTFE-GRE }\end{array}$} \\
\hline & Lesion & Brain & Contrast & Lesion & Brain & Contrast & \\
\hline 1 & 2025.5 & 609.6 & 1415.9 & 1726.6 & 515.3 & 1211.3 & 204.6 \\
\hline 2 & 1232.8 & 631 & 601.8 & 1113.2 & 531.7 & 581.5 & 20.3 \\
\hline 3 & 1504.1 & 507.1 & 997 & 892.7 & 406.6 & 486.1 & 510.9 \\
\hline 4 & 1196.6 & 507.1 & 689.5 & 819.3 & 406.6 & 412.7 & 276.8 \\
\hline 5 & 1091.1 & 727.5 & 363.6 & 682.4 & 531.9 & 150.5 & 213.1 \\
\hline 6 & 1320.8 & 891.8 & 429 & 887.1 & 558 & 329.1 & 99.9 \\
\hline 7 & 1183.3 & 906.4 & 276.9 & 855.6 & 576 & 279.6 & -2.7 \\
\hline 8 & 1254.1 & 793.3 & 460.8 & 712.5 & 587 & 125.5 & 335.3 \\
\hline 9 & 1209.9 & 793.3 & 416.6 & 844.2 & 587 & 257.2 & 159.4 \\
\hline 10 & 1919.4 & 770.9 & 1148.5 & 1189.6 & 476.9 & 712.7 & 435.8 \\
\hline 11 & 1508.9 & 857.5 & 651.4 & 970.3 & 658.6 & 311.7 & 339.7 \\
\hline 12 & 1329 & 626.1 & 702.9 & 1017.5 & 554.3 & 463.2 & 239.7 \\
\hline
\end{tabular}

The difference in the average values of 3D-IR-BTFE $3 \mathrm{~mm}$ and 3D-GRE $3 \mathrm{~mm}$ is shown in Figure 7. The difference test of the average value was $t(11)=5.247$, $\mathrm{p}<0.01(\mathrm{p}=0.00027), \mathrm{d}=0.74$. From this result, we can conclude that 3D-IR-BTFE has a better contrast than 3D-GRE.

\section{Discussion}

What is required in clinical diagnostic imaging of brain metastasis is to capture the presence of metastatic lesions, and the extent and size of metastatic lesions are important. If gadolinium contrast media is prescribed for the patient, I can reinforce contrast according to the T1 shortening effect. However, gadolinium contrast media and the $\mathrm{T} 1$ shortening effect are not necessarily in direct proportion. It is standard to prescribe $0.2 \mathrm{ml}$ per $1 \mathrm{~kg}$ of the patient's weight. For a brain metastasis lesion search, it is permitted to administer twice the amount of a specific gadolinium contrast agent only. The contrast enhancing effect of gadolinium contrast media has a limit. For this reason, we have to increase the contrast with the imaging method or static magnetic field strength. The factor that can raise the T1 contrast most in the imaging method is IR. 3D-IR-BTFE secured $\mathrm{T} 1$ contrast by adding IR.

In the image pick-up in two-dimensions, due to the characteristic of RF pulse, MRI equipment needs to prepare an interval between slices. This may be a challenge when searching for metastatic lesions-a challenge that could be solved if it could image in three dimensions. However, imaging in three dimensions increases imaging time. To eliminate this issue, a high signal-noise ratio imaging 


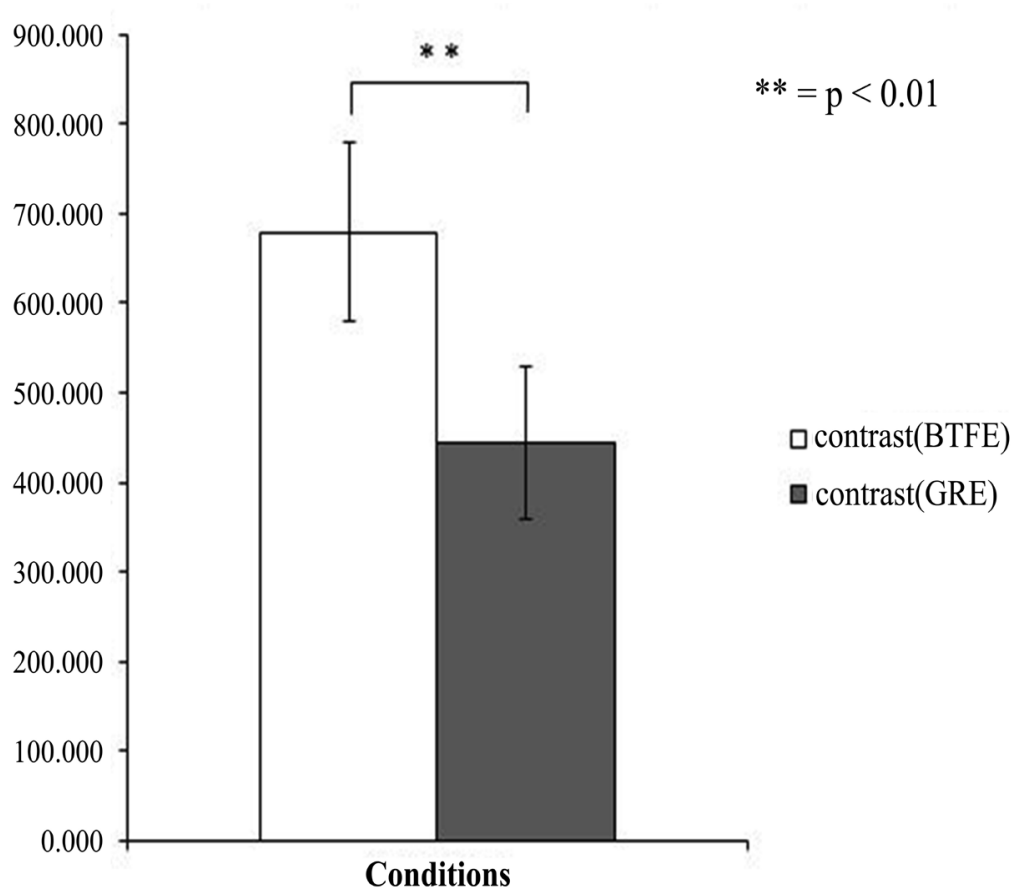

Figure 7. Shows the difference between the average values of 3D-IR-BTFE and 3D-GRE.

method must be selected. BTFE, in which TR is set short and imaged in a steady state without eliminating residual transverse magnetization, is an imaging method that can obtain excellent SNR and contrast. In the MRI apparatus studied here, the signal of the transition period can also be acquired. The signal of transition period can enlarge influence the T1 contrast. The T1 contrast can be increased by restoring longitudinal magnetization. To realize the longitudinal magnetization recovery, the parameter's shot interval was lengthened to $4000 \mathrm{~ms}$. I considered the possibility that the recovery of the longitudinal magnetization became larger by these, and excellent $\mathrm{T} 1 \mathrm{emphasis}$ was obtained. 3D-IR-BTFE considered SNR to be larger by BTFE than SE and gradient echo. It is likely that the good contrast was obtained by adding IR. To obtain further contrast, it is necessary to optimize IR and the numerical value of the shot interval.

Based on the above discussion, post-contrast 3D-IR-BTFE is superior to diagnose both brain metastatic lesions and the spread of metastatic lesions. It expected to be added to conventional SE-T1WI and 3D-GRE. From the restriction of SAR, the image pick-up is difficult with 3-Tesla equipment. In a high magnetic field device with 3-Tesla or more, it is necessary to investigate another imaging method that has IR added, and to a method of reducing the contrast medium.

The 3D-IR-BTFE method, which is a new imaging method developed this time, can achieve high contrast in a short time compared with the conventional imaging method by combining the BTFE method with a high SNR and the IR method for increasing the contrast It became clear from the research results. 


\section{Conclusion}

This research was presented in September 2009 at the 37th Japanese Society of Magnetic Resonance in Medicine convention. Since the use of gadolinium contrast media has become increasingly strict in recent years, I am reporting this research. This study found that 3D-IR-BTFE with IR added was superior in contrast. It is possible to acquire images of thin slices in a shorter time than with the conventional imaging method and the coherent-type gradient echo method could be used as a possible apparatus. To diagnose with the same degree of contrast as a spin echo or gradient echo, it is possible to reduce the contrast medium. The further examination is required for what contrast media loss in quantity is realized concretely. These results suggest that 3D-IR-BTFE, with excellent contrast, could be useful for other examinations at sites with little effect of motion.

\section{Conflicts of Interest}

The author indicated no conflicts of interest.

\section{References}

[1] Akeson, F., Larssqn, E., Kristoffersen, D., et al. (1995) Brain Metastases-Comparison of Gadodiamide Injection Enhanced MR Imaging at Standard and High Dose, Contrast-Enhanced CT and Non-Contrast-Enhanced MR Imaging. Acta Radiologica, 36, 300-306. https://doi.org/10.1177/028418519503600318

[2] Schorner, W., Laniado, M., Niendorf, H.P., et al. (1986) Time Dependent Changes in Image Contrast in Brain Tumors after Gadolinium-DTPA. AJNR, 7, 1013-1020.

[3] Russell, E.J., Geremia, G.K., Johnson, C.E., et al. (1987) Multiple Cerebral Metastases: Detectability with Gd-DTPA-Enhanced MR Imaging. Radiology, 165, 609-617. https://doi.org/10.1148/radiology.165.3.3317495

[4] Suzuki, K., Yamamoto, M., Hasegawa, Y., et al. (2004) Magnetic Resonance Imaging and Computed Tomography in the Diagnoses of Brain Metastases of Lung Cancer. Lung Cancer, 46, 357-360. https://doi.org/10.1016/j.lungcan.2004.05.011

[5] Kanda, T., Ishii, K., Kawaguchi, H., et al. (2013) High Signal Intensity in the Dentate Nucleus and Globus Pallidus on Unenhanced T1-Weighted MR Images: Relationship with Increasing Cumulative Dose of a Gadolinium-Based Contrast Material. Radiology, 270, 834-841. https://doi.org/10.1148/radiol.13131669

[6] Kanda, T., Osawa, M., Oba, H., et al. (2015) High Signal Intensity in Dentate Nucleus on Unenhanced T1-Weighted MR Images: Association with Linear versus Macrocyclic Gadolinium Chelate Administration. Radiology, 275, 803-809. https://doi.org/10.1148/radiol.14140364

[7] Kanda, T., Oba, H., Toyoda, K., et al. (2016) Brain Gadolinium Deposition after Administration of Gadolinium-Based Contrast Agents. Japanese Journal of Radiology, 34, 3-9. https://doi.org/10.1007/s11604-015-0503-5

[8] Brant-Zawadzki, M., Gillan, G.D. and Nitz, W.R. (1992) MP RAGE: A Three-Dimensional, T1-Weighted, Gradient-Echo Sequence-Initial Experience in the Brain. Radiology, 182, 769-775. https://doi.org/10.1148/radiology.182.3.1535892

[9] Huber, A., Schoenberg, S.O., Spannagl, B., et al. (2006) Single-Shot Inversion Re- 
covery True FISP for Assessment of Myocardial Infarction. AJR, 186, 627-633.

[10] Kanda, Y. (2013) Investigation of the Freely Available Easy-to-Use Software "EZR" for Medical Statistics. Bone Marrow Transplantation, 48, 452-458.

https://doi.org/10.1038/bmt.2012.244 